\title{
Geometric Hawkes Processes with Graph Convolutional Recurrent Neural Networks
}

\author{
Jin Shang, Mingxuan Sun \\ Division of Computer Science and Engineering \\ Louisiana State University \\ jshang2@1su.edu,msun@csc.lsu.edu
}

\begin{abstract}
Hawkes processes are popular for modeling correlated temporal sequences that exhibit mutual-excitation properties. Existing approaches such as feature-enriched processes or variations of Multivariate Hawkes processes either fail to describe the exact mutual influence between sequences or become computational inhibitive in most real-world applications involving large dimensions. Incorporating additional geometric structure in the form of graphs into Hawkes processes is an effective and efficient way for improving model prediction accuracy. In this paper, we propose the Geometric Hawkes Process (GHP) model to better correlate individual processes, by integrating Hawkes processes and a graph convolutional recurrent neural network. The deep network structure is computational efficient since it requires constant parameters that are independent of the graph size. The experiment results on real-world data show that our framework outperforms recent state-of-art methods.
\end{abstract}

\section{Introduction}

Hawkes processes, which are capable of modeling temporal events that exhibit self-exciting properties, have been widely applied in various applications such as supporting decision making in smart health (Xu et al. 2017), inferring granger causality (Xu, Farajtabar, and Zha 2016), and predicting recurrent user behaviors (Zhou, Zha, and Song 2013; Du et al. 2016; Shang and Sun 2018). Generally, Hawkes processes are useful for modeling a collection of correlated event sequences such as earthquakes at $N$ locations or the diffusion of $M$ infectious diseases among a group of $N$ people. For example, in analyzing on-line user behaviors such as visiting websites, recent approaches such as (Du et al. 2015) treat the recurrent events of each user-item pair as an onedimensional Hawkes process, and assume the parameters of all processes have a low-rank structure. However, methods that typically treat each process independently would fail to achieve good performance when there are insufficient observations for each process.

Multivariate Hawkes processes (Liniger 2009) are suitable for modeling multiple correlated sequences, where the occurrence of an event in one sequence may influence the

Copyright (C) 2019, Association for the Advancement of Artificial Intelligence (www.aaai.org). All rights reserved. occurrence of new events in another. For example, in social event analysis, the events of an individual user can be modeled as an one-dimensional Hawkes process and events in a network can be modeled as a Multivariate Hawkes process (Farajtabar et al. 2014; Mei and Eisner 2017; Yang et al. 2018), which captures the correlations of both endogenous and exogenous event intensities. Extensive studies (Lemonnier, Scaman, and Kalogeratos 2017; Etesami et al. 2016; Eichler, Dahlhaus, and Dueck 2017; Xu, Farajtabar, and Zha 2016) have focused on estimating the excitation matrix of multivariate processes for different inference tasks. However, those approaches are either unable to accurately capture the mutual influence between processes or become computational inhibitive in most real-world events involving large dimensions (Eichler, Dahlhaus, and Dueck 2017; Hall and Willett 2016).

Incorporating geometric structure in the form of graphs into Hawkes processes is an effective and efficient way for improving model prediction accuracy. In many real world applications, correlations between different Hawkes processes can be encoded by a graph. For example, in modeling the sequences of user-item interactions, the similarity of users and items can be represented by a user graph and an item graph, respectively. Such additional graph information can be used to impose smoothness priors on the parameters such as the base intensities of each individual process. Recently, geometric deep learning (Bruna et al. 2014; Defferrard, Bresson, and Vandergheynst 2016; Kipf and Welling 2017; Monti, Bronstein, and Bresson 2017) are promising techniques that can learn meaningful representations for geometric structure data such as graphs and have been successfully applied in various applications such as matrix completion.

In this paper, we propose a novel Geometric Hawkes Process (GHP) model by integrating geometric deep learning into Hawkes processes, which aims to efficiently capture meaningful patterns in a large collection of correlated sequences of recurrent events. Specifically, each sequence is modeled as a Hawkes process and the proximities between different processes are encoded in a graph. A novel convolutional and recurrent neural network is adopted to extract local meaningful patterns from the graph. The learned meaningful embeddings are then used to generate parameters such as the base intensities that characterize Hawkes processes. 
Comparing to traditional methods, our GHP correlates each individual Hawkes process effectively through graph embedding and it is computational efficient since the deep network structure requires constant parameters that are independent of the graph size. To the best of our knowledge, our GHP model is the first one to learn Hawkes processes with geometric deep learning. We also present the detail design of the single-graph and multi-graph cases for our Geometric Hawkes Process (GHP) model. Extensive experiments on real-world datasets demonstrate the predicting performance improvements of our model in comparison with the state of the art.

\section{Related Work}

Variations of Hawkes processes have been proposed for modeling correlated sequences. For example, the work by Zhou et al. (Zhou, Zha, and Song 2013) uses a multidimensional Hawkes process to learn the social interactivity in a sparse low-rank network. The work by Farajtabar et al. (Farajtabar et al. 2014) uses a Multivariate Hawkes process to model social events, which can capture both endogenous and exogenous event intensities. For modeling collections of user-item interactions, Du et al. (Du et al. 2015) assume that sequences of all user-item pairs are independent and the coefficients of all these point processes have low rank structure. A co-evolutionary latent feature process (Wang et al. 2016) has been further proposed to construct interdependent Hawkes processes by taking advantage of additional features such as user features, item features, and interaction features between users and items. Those features are globally embedded to Hawkes processes. However, those techniques do not fully exploit the local geometric structures of different processes in the form of graphs.

Recently, geometric deep learning becomes promising because the convolutional framework can be applied on nonEuclidean data, e.g, graphs, to extract important features. Some studies such as (Niepert, Ahmed, and Kutzkov 2016) focus on the vertex domain. However, it's hard to define an appropriate neighbor for each vertex and the extracted features sometimes are not representative especially on high dimensional data structure. Another way is to formulate graph convolutional on spectral domain (Bruna et al. 2014; Defferrard, Bresson, and Vandergheynst 2016; Kipf and Welling 2017; Monti, Bronstein, and Bresson 2017), which is the key concept underlying our work.

The first version of Graph Convolutional Network (GCN) (Bruna et al. 2014) contains $n$ convolutional kernel parameters, which is not only computational inhibitive but also lacks spatial localization. To solve these problems, ChebyNet (Defferrard, Bresson, and Vandergheynst 2016) uses Chebyshev polynomial localized filters to replace the diagonal matrix, which reduces the computation complexity from $\mathcal{O}\left(n^{2}\right)$ to $\mathcal{O}(n)$. Based on this type of framework, a lot of studies (Kipf and Welling 2017; Monti, Bronstein, and Bresson 2017) apply GCN to several specific tasks such as text classification, traffic forecasting, and matrix completion. The most closest ones to ours are the applications on matrix completion. However, their work mainly focus on modeling two dimensional data in the from of a $M$ by $N$ matrix without considering temporal dynamics.

\section{Model}

In this section, we introduce our Geometric Hawkes Process model.

\section{Background on Hawkes Processes}

A univariate Hawkes process is a self-exiting temporal point process and the realization of the process consists of a list of discrete temporal events $\mathcal{T}=\left\{t_{i}\right\}_{i=1}^{n}$. It is suitable for modeling the mutual excitation between events such as the occurrences of earthquakes at a particular location. The conditional intensity function that characterizes a Hawkes process is defined as:

$$
\lambda(t)=\eta+\alpha \sum_{t_{i} \in \mathcal{T}_{t}} \kappa_{\sigma}\left(t-t_{i}\right),
$$

where $\kappa_{\sigma}(t):=\exp (-t / \sigma)$ is an exponential kernel function that captures temporal dependencies, $\sigma$ is the bandwidth parameter of the kernel, $\eta \geq 0$ is the baseline intensity that captures the long-term incentive to generate events, $\alpha \geq 0$ is the coefficient that scales the influence of each previous event, and $\mathcal{T}_{t}=\left\{t_{i} \mid t_{i}<t\right\}_{i=1}^{n}$ denotes the history up to but not including time $t$.

Generally in real world applications, we would like to model a collection of correlated event sequences such as earthquakes at $N$ locations. Intuitively, each of the $N$ sequences can be modeled as a self-exciting Hawkes process:

$$
\lambda_{u}(t)=\boldsymbol{h}_{u}+\boldsymbol{a}_{u} \sum_{t_{j}^{u} \in \mathcal{T}_{t}^{u}} \kappa_{\sigma}\left(t-t_{j}^{u}\right),
$$

where $u=1, \ldots, N$ is the index of sequences such as $u^{t h}$ location, $\boldsymbol{h}$ and $\boldsymbol{a}$ are both vectors of size $N$ and their $u^{\text {th }}$ entries represent the non-negative base intensity and the selfexciting coefficient for $u^{t h}$ process respectively. The sequence $\mathcal{T}_{t}^{u}=\left\{t_{j}^{u} \mid t_{j}^{u}<t\right\}_{j=1}^{n}$ denotes the set of historic events of $u^{\text {th }}$ process up to but not including time $t$.

For events involving a pair of entities such as the interaction events between $M$ users and $N$ items (e.g., various infectious diseases among a group of people), the occurrences of interaction events between user $u$ and item $i$ can be modeled as following:

$$
\lambda_{(u, i)}(t)=\boldsymbol{H}_{u, i}+\boldsymbol{A}_{u, i} \sum_{t_{j}^{u, i} \in \mathcal{T}_{t}^{u, i}} \kappa_{\sigma}\left(t-t_{j}^{u, i}\right),
$$

where $\boldsymbol{H}$ denotes an $m \times n$ matrix with the $(u, i)^{t h}$ entry equal to the non-negative base intensity for pair $(u, i), \boldsymbol{A}$ denotes an $m \times n$ matrix with the $(u, i)^{t h}$ entry equal to the self-exciting coefficient for pair $(u, i)$, and the sequence $\mathcal{T}_{t}^{u, i}=\left\{t_{j}^{u, i} \mid t_{j}^{u, i}<t\right\}_{j=1}^{n}$ denotes the set of historic events of pair $(u, i)$ up to but not including time $t$.

However, treating each process independently would fail to achieve good performance when there are insufficient observations for each process. Incorporating correlations between processes such as location proximities and user/item 
similarities can improve the model prediction accuracy. The proximity between multiple Hawkes processes can be represented as an undirected weighted graph such as a proximity network of locations, a social network of users, and a network encoding item similarities.

\section{Background on Geometric Deep Learning}

Formally, an undirected weighted graph is denoted as $G=$ $(V, E, W)$, where $V$ is a finite set of $|V|=n$ vertices, $E$ is the set of edges and $W \in \mathbb{R}^{n \times n}$ is the adjacency matrix with entries $W_{i j}>0$ if $(i, j) \in E$. For each graph, a Laplacian matrix, which is an $n \times n$ symmetric positive-semidefinite matrix, can be constructed to reflect useful properties of a graph. Usually, the graph Laplacian is constructed as:

$$
L=I_{n}-D^{-1 / 2} W D^{-1 / 2},
$$

where $D \in \mathbb{R}^{n \times n}$ is the degree matrix with $D_{i i}=\sum_{j} W_{i j}$ and $I_{n}$ is the identity matrix.

Graph Convolution Network (GCN) Graph convolution is typically formulated in the spectral domain through graph Fourier transform (Mallat 1999). Specifically, a graph Laplacian $L$ admits a spectral eigendecomposition of the form $L=U \Lambda U^{\top}$, where $U=\left[u_{0}, \ldots, u_{n-1}\right] \in \mathbb{R}^{n \times n}$ is the orthonormal matrix and is the complete set of the orthonnormal eigenvectors $\left\{u_{l}\right\}_{l=0}^{n-1} \in \mathbb{R}^{n}$, and $\Lambda=$ $\operatorname{diag}\left(\left[\lambda_{0}, \ldots, \lambda_{n-1}\right]\right) \in \mathbb{R}^{n \times n}$ is the diagonal matrix with the associated ordered real nonnegative eigenvalues $\left\{\lambda_{l}\right\}_{l=0}^{n-1}$. In particular, eigenvectors are known as the Fourier atoms in classical harmonic analysis and eigenvalues are usually interpreted as the frequencies of the graph. Given a function $\boldsymbol{x}=\left(x_{0}, \ldots, x_{n-1}\right)^{\top} \in \mathbb{R}^{n}$ on the vertices of the graph, the graph Fourier transform on graph $G$ is defined as $\hat{\boldsymbol{x}}=\left(\hat{x}\left(\lambda_{0}\right), \ldots, \hat{x}\left(\lambda_{n-1}\right)\right)=U^{\top} \boldsymbol{x} \in \mathbb{R}^{n}$ and its inverse is $\boldsymbol{x}=U \hat{\boldsymbol{x}}$ (Shuman et al. 2013). Thus, the spectral convolutional of function $\boldsymbol{x}$ and convolutional kernel function $\boldsymbol{y}$ on graph $G$ is given by (Bruna et al. 2014):

$$
(\boldsymbol{x} \star \boldsymbol{y})_{G}=U \cdot \operatorname{diag}\left(\left[\hat{y}\left(\lambda_{0}\right), \ldots, \hat{y}\left(\lambda_{n-1}\right)\right]\right) \cdot U^{\top} \boldsymbol{x},
$$

where $\odot$ is the element-wise Hadamard product. It is worth mentioning that convolutions are by definition linear operators that diagonalize in the spectral domain, according to the definition of Discrete Fourier Transform and the Convolution Theorem (Mallat 1999). Thus, a GCN layer can be defined as $\boldsymbol{x}_{\text {output }}=\sigma\left((\boldsymbol{x} \star \boldsymbol{y})_{G}\right)$, where $\operatorname{diag}\left(\left[\hat{y}\left(\lambda_{0}\right), \ldots, \hat{y}\left(\lambda_{n-1}\right)\right]\right)$ represents parameters of learnable filters in the spectral domain, and $\sigma$ denotes the activation function (e.g. ReLU) which is applied on the vertexwise function values.

In order to reduce the computational complexity and the number of the parameters, as well as adding localization which is common in graph signal processing (Hammond, Vandergheynst, and Gribonval 2011), a polynomial filter was introduced by (Defferrard, Bresson, and Vandergheynst 2016). Thus, the GCN layer with one filter has the following forms: $\boldsymbol{x}_{\text {output }}=\sigma\left(\sum_{k=0}^{K-1} \theta_{k} L^{k} \boldsymbol{x}\right)$, where $\boldsymbol{\theta}=\left\{\theta_{k}\right\}_{k=0}^{K-1}$ is a vector of polynomial coefficients for such a filter and the number of parameters is $K$. Note that the formula involves only the computation of the Laplacian $L$ without the computation of its decomposition of $U$. Specifically, the filter can be approximated by the Chebyshev polynomial basis $T_{k}$ of degree $k$ (Hammond, Vandergheynst, and Gribonval 2011), where $T_{k}\left(\tilde{\lambda}_{l}\right)=2 \tilde{\lambda}_{l} T_{k-1}\left(\tilde{\lambda}_{l}\right)-T_{k-2}\left(\tilde{\lambda}_{l}\right)$ is defined in a recursive way with $T_{0}=1$ and $T_{1}=\tilde{\lambda}_{l}$. Thus, the GCN layer with one filter becomes (Defferrard, Bresson, and Vandergheynst 2016):

$$
\boldsymbol{x}_{\text {output }}=\sigma\left(\sum_{k=0}^{K-1} \theta_{k} T_{k}(\tilde{L}) \boldsymbol{x}\right),
$$

where $\tilde{L}=2 L / \lambda_{\max }-I_{n}$ is the rescaled Laplacian with scaled eigenvalues $\tilde{\Lambda}=2 \Lambda / \lambda_{\max }-I_{n}$ in the interval $[-1,1]$.

By applying kernel polynomial localization, the computational complexity becomes $\mathcal{O}(n)$ rather than $\mathcal{O}\left(n^{2}\right)$ (Defferrard, Bresson, and Vandergheynst 2016), as we don't need to do eigendecomposition. Also, the parameter number is only $K$ rather than $n$, and the convolutional kernel with spatial localization will benefit local feature extraction. A simplified variant of this filter (Kipf and Welling 2017), which assumes $K=1$ and $\lambda_{\max }=2$, also achieves good performance on classification tasks.

GCN with Multi-graph (Multi-GCN) According to the definition of multidimensional Fourier Transform, Graph Fourier Transform and GCN layers can be extended to multigraph version (Kurokawa, Oki, and Nagao 2017; Monti, Bronstein, and Bresson 2017). Given two scaled graph Laplacian (referred to single-graph convolutional layer) $L_{r} \in \mathbb{R}^{m \times m}$ and $L_{c} \in \mathbb{R}^{n \times n}$ with $m$ vertices on the row graph $G_{r}$ and $n$ vertices on the column graph $G_{c}$, a multiGCN layer with one filter is defined as (Monti, Bronstein, and Bresson 2017):

$$
\boldsymbol{X}_{\text {output }}=\sigma\left(\sum_{k=0}^{K-1} \sum_{k^{\prime}=0}^{K-1} \theta_{k k^{\prime}} T_{k}\left(\tilde{L}_{r}\right) \boldsymbol{X} T_{k^{\prime}}\left(\tilde{L}_{c}\right)\right)
$$

where function $\boldsymbol{X} \in \mathbb{R}^{m \times n}$ is two dimensional and such filter is parameterized by a $K \times K$ matrix of polynomial coefficients $\boldsymbol{\Theta}=\left(\theta_{k k^{\prime}}\right)$.

Generalized GCN Layers More generally, considering the computation effectiveness of convolution, we give the following generalized form of GCN layers, which is an high performance GCN layer referring to (Chellapilla, Puri, and Simard 2006) and convolution implementation in Caffe. Given $C$ input channels of $\left\{\boldsymbol{x}_{c}\right\}_{c=1}^{C}$ (a matrix of size $m \times C$ ) and $C^{\prime}$ output channels (output feature map size or the number of filters), the single-GCN layer has the generalized form:

$$
\boldsymbol{x}_{c_{\text {output }}^{\prime}}=\sigma\left(\sum_{c=1}^{C} \sum_{k=0}^{K-1} \theta_{k c, c^{\prime}} T_{k}(\tilde{L}) \boldsymbol{x}_{c}\right) .
$$

where $c^{\prime}=1, \ldots, C^{\prime}$.

Similarly, this can also be applied to multi-GCN layer. Given $C$ input channels of $\left\{\boldsymbol{X}_{c}\right\}_{c=1}^{C}$ (a tensor of size $m \times$ 
$n \times C$ ) and $C^{\prime}$ output channels, the multi-GCN layer has the generalized form:

$$
\boldsymbol{X}_{c_{\text {output }}^{\prime}}=\sigma\left(\sum_{c=1}^{C} \sum_{k=0}^{K-1} \sum_{k^{\prime}=0}^{K-1} \theta_{k k^{\prime} c, c^{\prime}} T_{k}\left(\tilde{L}_{r}\right) \boldsymbol{X}_{c} T_{k^{\prime}}\left(\tilde{L}_{c}\right)\right),
$$

Integration of GCN and RNN Furthermore, a GCN network coupled with a RNN network can progressively reconstruct the parameters and it has demonstrated to be highly efficient (Monti, Bronstein, and Bresson 2017). Specifically, the input of the GCN network is the original matrix $\boldsymbol{X}^{(0)}$. The output of the GCN network such as $C^{\prime}$ matrices are the input to a RNN network such as LSTM (Hochreiter and Schmidhuber 1997). Then, the output of the RNN network are the input to a fully connected layer to calculate the changes $\boldsymbol{d} \boldsymbol{X}$ of the input matrix $\boldsymbol{X}$. After several iterations (e.g. $T$ steps), the predicted value becomes $\boldsymbol{X}^{(T)}=$ $\boldsymbol{X}^{(T-1)}+\boldsymbol{d} \boldsymbol{X}^{(T-1)}$.

\section{Our Geometric Hawkes Processes (GHP)}

We propose a novel Geometric Hawkes Process (GHP) model by integrating the geometric deep learning into Hawkes processes, which aims to efficiently capture meaningful patterns in a large collection of correlated sequences of recurrent events. In our framework, each sequence is modeled as a Hawkes process and the proximities between different processes are encoded in graphs. Specifically, we propose two types of GHP: single-graph GHP and multigraph GHP. Single-graph GHP is particularly useful for modeling sequences with one type of graph such as modeling earthquakes at $N$ locations with a proximity network of locations. Multi-graph GHP is particularly useful for modeling sequences with multiple graphs such as modeling the diffusion of various infectious diseases among a group of people, where the relationship of people and diseases can be represented by a user graph and an item graph, respectively. The learned meaningful embeddings from graphs are then used to generate parameters such as the base intensities that characterize Hawkes processes.

Specifically, the parameters of single-graph GHP are $\boldsymbol{h}$, $\boldsymbol{a}$ as described in eq. (2) and they are functions defined on a graph, e.g., a user graph. Similarly, the parameters of multi-graph GHP are $\boldsymbol{H}$ and $\boldsymbol{A}$ as described in eq. (3), and they are functions defined on multiple graphs, e.g., a user graph and an item graph. The parameters are random initialized as $\boldsymbol{x}$ or $\boldsymbol{X}$ in equations eq. (6) and eq. (7) respectively, and will be optimized in deep geometric learning. The loss function is defined as the log-likelihood of observing the sequences of events. Formally, based on the survival analysis theory (Aalen, Borgan, and Gjessing 2008), the likelihood of observing a sequence of events $\mathcal{T}=\left\{t_{i}\right\}_{i=1}^{n}$ is $\prod_{t_{i} \in \mathcal{T}} \lambda\left(t_{i}\right) \cdot \exp \left(-\int_{0}^{\Gamma} \lambda(\tau) d(\tau)\right)$, where $\Gamma$ is the total observation time. We present the details for the two types of GHP as the following.

Single-graph GHP Specifically, for a collection of Hawkes processes according to eq. (2) and eq. (6), let $\mathcal{T}^{u}$ be the set of events induced by vertex $u=1, \ldots, m$. The log-likelihood of observing each sequence $\mathcal{T}^{u}$ is:

$$
\mathcal{L}\left(\mathcal{T}^{u} \mid \boldsymbol{x}_{\boldsymbol{\theta}, \boldsymbol{\zeta}}^{(T)}\right)=\sum_{t_{j}^{u} \in \mathcal{T}^{u}} \log \left(\boldsymbol{x}_{u}^{(T)} \Phi_{j}^{u}\right)-\boldsymbol{x}_{u}^{(T)} \Psi^{u},
$$

where:

$$
\begin{aligned}
\boldsymbol{x}_{u}^{(T)} & =\left(\boldsymbol{h}(u)^{(T)}, \boldsymbol{a}(u)^{(T)}\right), \\
\Phi_{j}^{u} & =\left(1, \sum_{t_{k}^{u}<t_{j}^{u}} \kappa_{\sigma}\left(t_{j}^{u}-t_{k}^{u}\right)\right)^{\top}, \\
\Psi^{u} & =\left(\Gamma, \sum_{t_{j}^{u} \in \mathcal{T}^{u}} \int_{t_{j}^{u}}^{\Gamma} \kappa_{\sigma}\left(t-t_{j}^{u}\right) d t\right)^{\top} .
\end{aligned}
$$

It is worth mentioning that the notation $\boldsymbol{x}_{\boldsymbol{\theta}, \boldsymbol{\zeta}}{ }^{(T)}$ emphasize the matrix depends on the parameters of GCN (polynomial coefficients $\boldsymbol{\theta}$ ) and those of the LSTM network (denote as $\zeta$ ) after $T$ steps. As a result, the log-likelihood of observing all vertices' sequences $\mathcal{O}=\left\{\mathcal{T}^{u}\right\}_{u}$ is a summation of terms by $\mathcal{L}(\mathcal{O})=\sum_{\mathcal{T}^{u} \in \mathcal{O}} \mathcal{L}\left(\mathcal{T}^{u}\right)$. Also, we want the variables $\boldsymbol{h}$ and $\boldsymbol{a}$ to be faithful to the graph structure $G$ with $m$ vertices and the corresponding graph Laplacian $L_{m \times m}$. Thus, we can add the graph regularizer $h\left(\boldsymbol{x}_{\boldsymbol{\theta}, \boldsymbol{\zeta}}\right)=$ $\rho\left\{\operatorname{tr}\left(\boldsymbol{h}^{\top} L \boldsymbol{h}\right)+\operatorname{tr}\left(\boldsymbol{a}^{\top} L \boldsymbol{a}\right)\right\}$ and the squared Frobenius norm $g\left(\boldsymbol{x}_{\boldsymbol{\theta}, \boldsymbol{\zeta}}\right)=\gamma\|\boldsymbol{h}\|_{F}^{2}+\beta\|\boldsymbol{a}\|_{F}^{2}$ as (Rao et al. 2015). Finally, we can obtain $\boldsymbol{h}$ and $\boldsymbol{a}$ by minimizing the following objective function:

$$
\begin{aligned}
O P T= & \min _{\boldsymbol{\theta}, \boldsymbol{\zeta}}-\frac{1}{|\mathcal{O}|} \sum_{\mathcal{T}^{u} \in \mathcal{O}} \mathcal{L}\left(\mathcal{T}^{u} \mid \boldsymbol{x}_{\boldsymbol{\theta}, \boldsymbol{\zeta}}{ }^{(T)}\right)+h\left(\boldsymbol{x}_{\boldsymbol{\theta}, \boldsymbol{\zeta}}{ }^{(T)}\right)+g\left(\boldsymbol{x}_{\boldsymbol{\theta}, \boldsymbol{\zeta}}{ }^{(T)}\right) \\
& \text { s.t. } \boldsymbol{x}_{\boldsymbol{\theta}, \boldsymbol{\zeta}}{ }^{(T)} \geq \mathbf{0}
\end{aligned}
$$

where $\boldsymbol{x}_{\boldsymbol{\theta}, \boldsymbol{\zeta}}=[\boldsymbol{h} ; \boldsymbol{a}]$, and $\rho \gamma, \beta$ control the trade-off between the constrains. After the parameters converging to optimal, we can directly use $x$ and eq. (2) to compute the intensity and make predictions.

Multi-graph GHP Similarly, we can give the objective function of multi-graph GHP. According to eq. (3) and eq. (7), let $\mathcal{T}^{u, i}$ be the set of events induced between vertex $u=1, \ldots, m$ and vertex $i=1, \ldots, n$. The log-likelihood of observing each sequence $\mathcal{T}^{u, i}$ is:

$$
\mathcal{L}\left(\mathcal{T}^{u, i} \mid \boldsymbol{X}_{\Theta, \zeta}^{(T)}\right)=\sum_{t_{j}^{u, i} \in \mathcal{T}^{u, i}} \log \left(\boldsymbol{X}_{u, i}^{(T)} \Phi_{j}^{u, i}\right)-\boldsymbol{X}_{u, i}^{(T)} \Psi^{u, i}
$$

where:

$$
\begin{aligned}
\boldsymbol{X}_{u, i}^{(T)} & =\left(\boldsymbol{H}(u, i)^{(T)}, \boldsymbol{A}(u, i)^{(T)}\right), \\
\Phi_{j}^{u, i} & =\left(1, \sum_{t_{k}^{u, i}<t_{j}^{u, i}} \kappa_{\sigma}\left(t_{j}^{u, i}-t_{k}^{u, i}\right)\right)^{\top}, \\
\Psi^{u, i} & =\left(\Gamma, \sum_{t_{j}^{u, i} \in \mathcal{T}^{u, i}} \int_{t_{j}^{u, i}}^{\Gamma} \kappa_{\sigma}\left(t-t_{j}^{u, i}\right) d t\right)^{\top} .
\end{aligned}
$$

In this case, the notation $\boldsymbol{X}_{\Theta, \zeta}{ }^{(T)}$ emphasize the matrix depends on the parameters of multi-GCN (polynomial coefficients $\Theta$ ) and those of the LSTM network (denote as $\zeta$ ) after $T$ steps. Similarly, the log-likelihood of observing all vertices' sequences $\mathcal{O}=\left\{\mathcal{T}^{u, i}\right\}_{u, i}$ is a summation of 


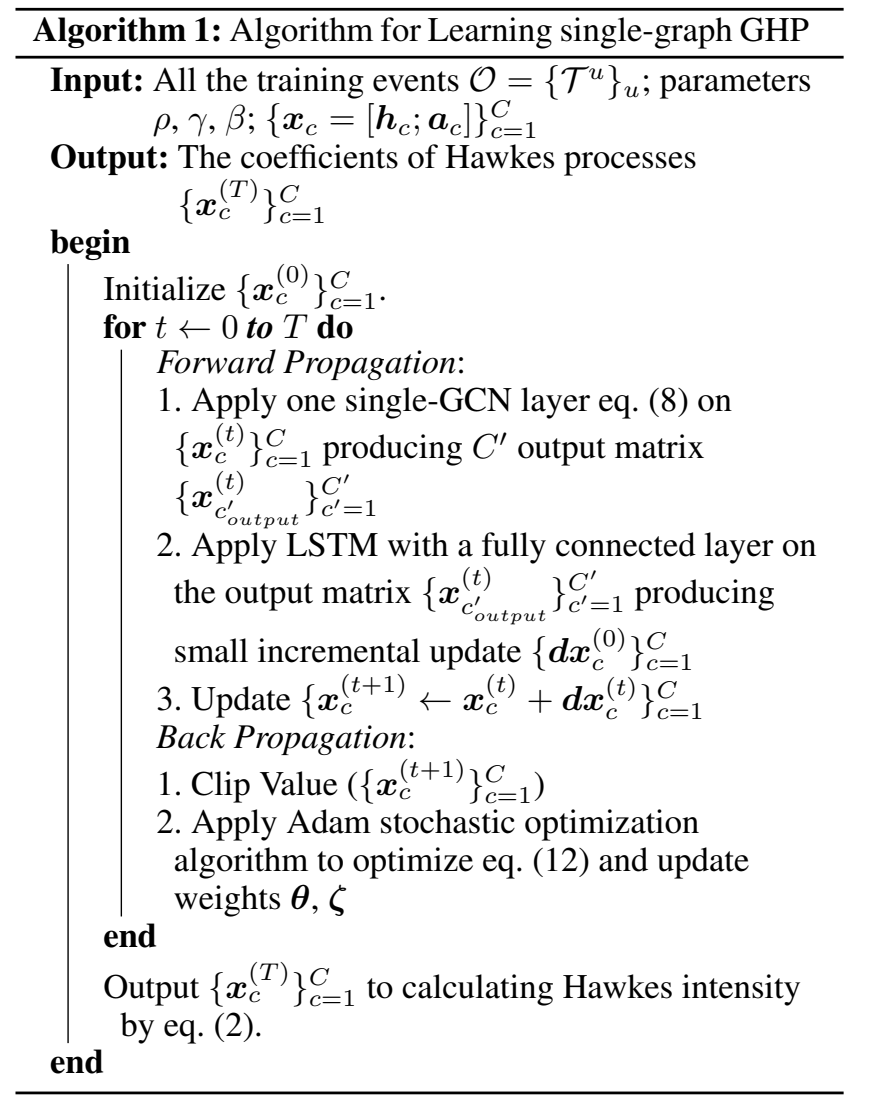

terms by $\mathcal{L}(\mathcal{O})=\sum_{\mathcal{T} u, i \in \mathcal{O}} \mathcal{L}\left(\mathcal{T}^{u, i}\right)$. Given the row graph structure $G_{r}$ with $m$ vertices and the column graph structure $G_{c}$ with $n$ vertices, the corresponding graph Laplacian are $L_{r} \in \mathbb{R}^{m \times m}$ and $L_{c} \in \mathbb{R}^{n \times n}$. Thus, we can add the multi-graph regularizers as $\tilde{h}\left(\boldsymbol{X}_{\boldsymbol{\Theta}, \boldsymbol{\zeta}}\right)=\rho\left\{\operatorname{tr}\left(\boldsymbol{H}^{\top} L_{r} \boldsymbol{H}\right)+\right.$ $\left.\operatorname{tr}\left(\boldsymbol{H} L_{c} \boldsymbol{H}^{\top}\right)+\operatorname{tr}\left(\boldsymbol{A}^{\top} L_{c} \boldsymbol{A}\right)+\operatorname{tr}\left(\boldsymbol{A} L_{c} \boldsymbol{A}^{\top}\right)\right\}$ (Kalofolias et al. 2014). It is worth mentioning that two matrix with $m \times n$ dimension contain too many parameters. Usually, a lot of points' attributes can be categorized into a limited number of types for the real world data. So, we assume $\boldsymbol{H}$ and $\boldsymbol{A}$ have low-rank structures, and we can add the nuclear norm $\tilde{g}\left(\boldsymbol{X}_{\Theta, \zeta}\right)=\gamma\|\boldsymbol{H}\|_{*}+\beta\|\boldsymbol{A}\|_{*}$ as (Du et al. 2015), which is frequently used as a convex surrogate penalty term for matrix rank. Finally, we can obtain $\boldsymbol{H}$ and $\boldsymbol{A}$ by minimizing the following objective function:

$$
\begin{aligned}
O P T= & \min _{\boldsymbol{\Theta}, \boldsymbol{\zeta}}-\frac{1}{|\mathcal{O}|} \sum_{\mathcal{T}} \mathcal{L}\left(\mathcal{T}^{u, i} \in \boldsymbol{X}_{\Theta, \zeta}{ }^{(T)}\right)+\tilde{h}\left(\boldsymbol{X}_{\Theta, \zeta}{ }^{(T)}\right)+\tilde{g}\left(\boldsymbol{X}_{\Theta, \zeta}{ }^{(T)}\right) \\
& \text { s.t. } \boldsymbol{X}_{\Theta, \zeta}{ }^{(T)} \geq \mathbf{0},
\end{aligned}
$$

where $\boldsymbol{X}_{\boldsymbol{\Theta}, \boldsymbol{\zeta}}=[\boldsymbol{H} ; \boldsymbol{A}]$, and $\rho \gamma, \beta$ control the trade-off between the constrains. After the parameters converging to optimal, we can directly use $\boldsymbol{X}$ and eq. (3) to compute the intensity and make predictions.

Learning with Clipping We can use several stochastic optimization algorithms such as SGD and Adam (Kingma and $\mathrm{Ba} 2015$ ) to solve the log-likelihood with regularizers. However, as Hawkes processes have non-negative parameters, the objective function should be optimized under such non-negative constraints eqs. (12) and (15). Since it is the inequality constraints, directly solving it by adding Lagrange multiplier or Kuhn-Tucker method (Wallace 2004) will introduce the Complementary Slackness Conditions, which makes it more complex. To enforce the non-negative constraints on the objective function, we clip the value to lie within a compact space after each temporal step $t=0, \ldots, T$ and make the lower bound greater than zero. We present the following learning algorithms 1 and 2 for single-graph and multi-graph GHP, respectively.

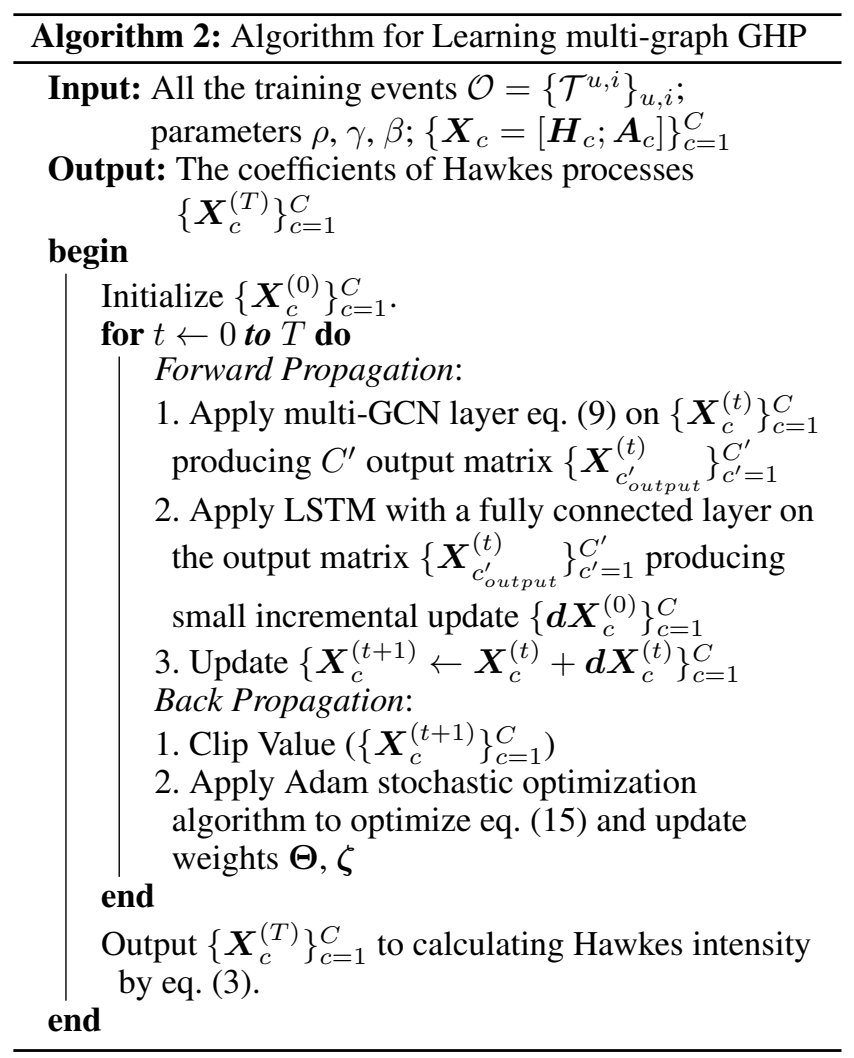

Computational Complexity By applying polynomial localization, the single-GCN eq. (6) reaches $\mathcal{O}(n)$ (Defferrard, Bresson, and Vandergheynst 2016) rather than using eq. (7) with complexity $\mathcal{O}\left(n^{2}\right)$, where $n$ is the number of vertices of the graph. Thus, the multi-GCN has the complexity of $\mathcal{O}(m n)$ (Monti, Bronstein, and Bresson 2017) considering $C, C^{\prime}, K \ll \min (m, n)$. Also, the learning complexity of LSTM network is $\mathcal{O}(\boldsymbol{W})$, where the number of parameters $\boldsymbol{W}=4 n_{c}^{2}+4 n_{c} n_{i}+n_{c} n_{o}+3 n_{c}$ (Sak, Senior, and Beaufays 2014), and the number of memory units, input units and output units are equal to the number of output feature map size of the GCN $n_{c}=n_{i}=n_{c}=C^{\prime}$ in our network. As a result, such single-GCN + RNN network has the complexity of $\mathcal{O}\left(n+n \cdot C^{\prime} \cdot C^{\prime}\right)=\mathcal{O}(n)$ per time step and the multigraph one has the similar complexity of $\mathcal{O}(m n)$ per time step. It is worth mentioning that these are computed globally. To make it more efficient, we can also address several mini-batch with $P$ samples from $n$ or $m n$, which makes the algorithm independent of the graph size and achieve $\mathcal{O}(P)$ complexity. 


\section{Experiment and Results}

In this section, we introduce the experiments.

\section{Experimental Settings and Evaluation Metrics}

We evaluate our model on three real world datasets which contain temporal interactions between a set of users and a set of items. Specifically, the IPTV dataset (Xu, Farajtabar, and Zha 2016) contains 7100 users and 436 TV programs with some program features such as genres. For each user-item pair, it contains a sequence of viewing time during the period of January to November 2012. The Yelp ${ }^{1}$ dataset is available from Yelp dataset challenge. After pre-processing, it records the time of writing reviews for $17 \mathrm{k}$ businesses by 100 users during a period of 11 years. The Reddit ${ }^{2}$ dataset contains the time of posting discussions between random selected 1000 users and 1403 threads in January 2014.

As suggested in (Monti, Bronstein, and Bresson 2017), a user or item graph can be constructed as an unweighted $k$ nearest neighbor graph in the space of features such as TV features. In cases where user and item features are not available, we can construct a two-dimensional user-item matrix from the time sequences where each entry indicates the total count of user-item interactions, and apply SVD to get a latent feature vector for each use or item. We can model these datasets using either single-graph GHP or multi-graph GHP. For the first case, the parameters are regraded as vector functions on a graph (e.g., user graph) and the values of each dimension (e.g., item index) are regraded as different channels. For the second case, the parameters are regraded as scalar functions on both user and item graphs and the size of the input channel is one.

There are three metrics to evaluate the performance of the model. In the experiments, we use the events before time $T \cdot p$ as the training data, and the rest of them as testing data, where $T$ is the length of the total time, and $p=0.76$ is the proportion where we split the data.

Test Loss: It is defined as in the objective function eqs. (12) and (15) with fixed coefficients of Hawkes processes learned using events in the training set.

Item Relevance: Given the history $\mathcal{T}=\left\{t_{i}\right\}_{i=1}^{n}$ of a specific user $u$, we calculate the survival rates for all the items at each testing time $t$, that is $S_{i}(t)=\exp \left(-\int_{t_{n}^{i}}^{t} \lambda_{i}(\tau) d(\tau)\right)$. We then order all the survivals and compute the rank of the ground-truth item the user interacts at testing time $t$. Ideally the ground-truth item should be ordered at rank one. Following (Wang et al. 2016), we report mean average rank (MAR) of all testing cases. A smaller value of MAR indicates better predictive performance.

Time Prediction Accuracy: Given a specific pair of user $u$ and the item $i$, we record the mean absolute error (MAE) of the next predicted time and the ground truth of testing time $t$. The predicted time is calculated by the density of next event time as $f(t)=\lambda_{(u, i)}(t) S_{(u, i)}(t)$, and then use the expectation to predict the next event. Furthermore, we also give the relative percentage of the prediction error (Err $\%)$.

\footnotetext{
${ }^{1}$ https://www.yelp.com/dataset/challenge

${ }^{2}$ https://dynamics.cs.washington.edu/data.html
}

\section{Baseline Methods}

Po: Poisson processes are simplified Hawkes processes without capturing temporal dependencies. The only parameter to characterize Poisson is the base intensity $\eta$, which is a constant.

Po-T: Poisson-Tensor uses Poisson regression error instead of RMSE as the loss function when fitting the data. The intensity are regarded as the number of events in each discretized time slot (Chi and Kolda 2012). It assumes that the missing values are not random, and thus simulating the values with Poisson distribution is more reasonable than with Gaussian. Once we get the model parameters, there are two ways to simulate the intensity of test data. One is using the intensity that we have got only in the last time interval, and the other is using the average intensity of all the training time intervals. We report the best performance of these two choices.

LRH: LowRankHawkes is a collection of Hawkes processes (Du et al. 2015) assuming that all processes are independent and the parameters are low rank matrices. However, there are no interactions between different processes.

Coevol: Coevolve is a co-evolutionary latent feature process (Wang et al. 2016) which constructs interdependent Hawkes processes by embedding user and item features globally into each process. This method actually combines all events happening before the current event from different processes when fitting the parameter of each individual process. However, the performance in terms of item relevance may be affected due to unrelated events. In addition, if no features are used, the model reduces to a Poisson process.

\section{Compare Ours of Different Parameters}

We first investigate the influence of important parameters in our GHP model by evaluating them using the testing loss. Specifically, the parameters are types of graph, k-nearest neighbor, and finally the deep learning architectures.

Single vs Multi-graph We show the results of testing losses with multi-graph input compared with only singlegraph input, e.g. only a user graph or an item graph, of IPTV dataset in fig. 1(a). As we can see, the testing loss with multigraph input outperforms that with only single-graph input, which prove that the graph information is extracted well by the GCN + LSTM networks. It is worth mentioning that the IPTV dataset contains 7100 users and 436 TV show items, so using only the user graph achieves better results than using only the item graph. Also, the testing loss shows that the less information inputed, the faster it overfits the data.

Number of K-neighbors We also investigate the number $\mathrm{K}$ 's effect when constructing the K-nearest neighbor graph. In fig. 1(b), we present the testing losses of IPTV dataset with 2, 5, 10, 15, 20 -NN graph input of multi-graph GHP model. The figure demonstrates that give the $\mathrm{K}$ in a reasonable range, we can achieve a stable and accurate estimation of the model. The results show that $k=10$ is the best for IPTV dataset. In the experiment, we use the same $\mathrm{K}$ for both user and item input graphs. However, we can separately set $\mathrm{K}$ for the user graph and the item graph to make it more 


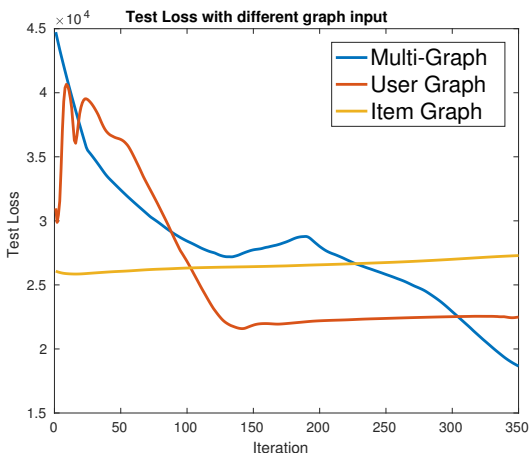

(a) Graph input

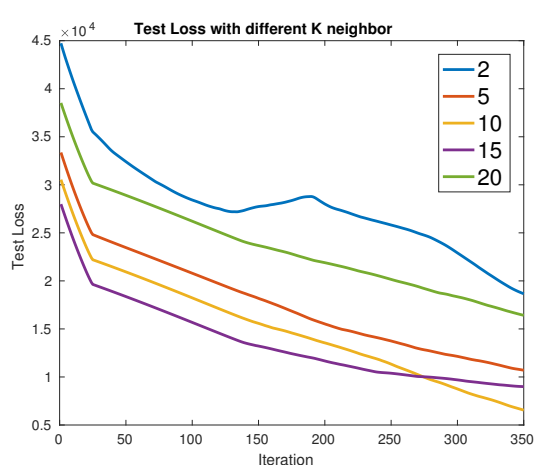

(b) K neighbor

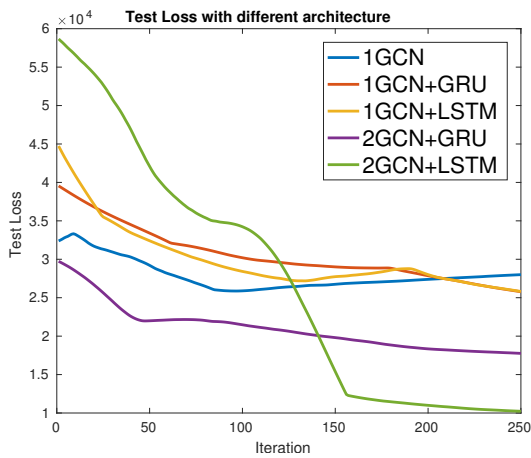

(c) Architecture

Figure 1: Testing loss with respect to different graph inputs, different number of neighbors, and architectures on IPTV data.

flexible. According to fig. 1(a) and fig. 1(b), our GHP model benefits from the input graph information and extracts useful features from these interactions, and thus the model overcomes the isolation of point process models such as (Du et al. 2015).

Variations of Architecture Setting We compare different architecture settings of our model and the results are presented in fig. 1(c). First of all, the RNN structure such as LSTM or GRU is essential to learn the diffusion process of coefficients. The LSTM is more effective compared to GRU (Chung et al. 2014) because LSTM can remember more historical information. Besides, the results show that adding more GCN layers enhances the performance of modeling Hawkes processes, which indicates that deeper network may extract more useful features. As data size increases, it is necessary to build deeper architectures. More extensive studies on the architecture of GCN in different applications can be found at (Kipf and Welling 2017; Monti, Bronstein, and Bresson 2017). In our experiment, we found the structure of two GCN layers plus one LSTM layer works best.

Table 1: Average prediction performance comparison on IPTV, Yelp, and Reddit datasets.

\begin{tabular}{ccccccc}
\hline \multirow{2}{*}{ Datasets } & \multirow{2}{*}{ Metrics } & \multicolumn{5}{c}{ Methods } \\
\cline { 3 - 7 } & & Our & LRH & Coevol & Po & Po-T \\
\hline \multirow{3}{*}{ IPTV } & MAR & $\mathbf{1 . 6 4 3}$ & 5.175 & 13.57 & 173.7 & 178.7 \\
& MAE & 361.0 & 822.1 & $\mathbf{1 6 0 . 3}$ & 993.1 & 933.6 \\
& Err \% & 5.13 & 12.27 & $\mathbf{2 . 3 5}$ & 14.83 & 13.89 \\
\hline \multirow{3}{*}{ Yelp } & MAR & $\mathbf{9 4 . 6 2}$ & 116.0 & 671.2 & 7778 & 1738 \\
& MAE & $\mathbf{4 9 9 . 0}$ & 845.7 & 587.3 & 850.9 & 587.1 \\
& Err \% & $\mathbf{1 4 . 5 9}$ & 23.71 & 17.49 & 23.91 & 17.48 \\
\hline \multirow{2}{*}{ Reddit } & MAR & $\mathbf{6 . 0 1 0}$ & 49.14 & 82.44 & 128.2 & 85.49 \\
& MAE & 5367 & 8476 & $\mathbf{5 3 2 3}$ & 10314 & 9155 \\
& Err \% & $\mathbf{1 4 . 1 5}$ & 21.50 & 14.27 & 26.59 & 24.09 \\
\hline
\end{tabular}

\section{Compare with Baselines}

We compare our GHP model with some state-of-art baselines by evaluating the metrics of item relevance and time prediction accuracy as shown in table 1 . We use multi-graph
GHP model and the results show that our method outperforms other baseline methods in general. For IPTV and Reddit datasets, the exception occurs on time prediction of $\mathrm{Co}$ evol. Specifically, the Coevol method uses a weighted summation of all the events happened before the current event to simulate one point's intensity. Therefore, the returning-time prediction is good since a large number of events are used to simulate the intensity function. The embedding of auxiliary features such as TV genres is also helpful in improving prediction accuracy. However, the item relevance prediction becomes worse (Wang et al. 2016) because the parameters of the individual process are influenced by unrelated processes. Meanwhile, we can see that the Hawkes process based models, such as our model, Coevol, and $L R H$, get better performances when there are sufficient history events (with nearly 400 events per point for IPTV and 30 events for Reddit) in comparison with the Poisson related models. For Yelp data, as each point process only has fewer than 3 events in average, the time prediction is similar among $L R H$ and $P o$, which means that the history is not such an important factor. In this time sparsity case, factorization model $\mathrm{Po}-\mathrm{T}$ gets better results than point process based models. For all three datasets, $L R H$ with low-rank assumption, performs worse than our GHP that integrates graphs with low rank assumption. Obviously, integrating graphs can better capture the correlations between different processes.

\section{Conclusions}

In this paper, we present a novel framework that integrates the graph convolutional recurrent neural network and Hawkes processes to model temporal events. Our model can be applied to a collection of correlated temporal sequences of recurrent events, and it is able to correlate each sequence through graph embedding. We also present single-graph and multi-graph settings of our model. Extensive experiments on real-world datasets demonstrate the performance improvements of our model in comparison with the state of the art. Future work includes integrating Hawkes Processes with other different types of deep neural network structures and extending to other applications. 


\section{Acknowledgement}

This work was supported in part by the Louisiana Board of Regents under Grant LEQSF(2017-20)-RD-A-29. The authors would also like to thank Yichen Wang and Le Song from Georgia Tech for their helpful discussions.

\section{References}

Aalen, O.; Borgan, O.; and Gjessing, H. 2008. Survival and Event History Analysis: A Process Point of View. Springer Science \& Business Media.

Bruna, J.; Zaremba, W.; Szlam, A.; and LeCun, Y. 2014. Spectral networks and locally connected networks on graphs. In Proc. International Conference on Learning Representations (ICLR).

Chellapilla, K.; Puri, S.; and Simard, P. 2006. High performance convolutional neural networks for document processing. In Tenth International Workshop on Frontiers in Handwriting Recognition.

Chi, E. C., and Kolda, T. G. 2012. On tensors, sparsity, and nonnegative factorizations. SIAM Journal on Matrix Analysis and Applications 33(4):1272-1299.

Chung, J.; Gulcehre, C.; Cho, K.; and Bengio, Y. 2014. Empirical evaluation of gated recurrent neural networks on sequence modeling. arXiv preprint arXiv:1412.3555.

Defferrard, M.; Bresson, X.; and Vandergheynst, P. 2016. Convolutional neural networks on graphs with fast localized spectral filtering. In Proc. of the Annual Conference on Neural Information Processing Systems (NIPS), 3844-3852.

Du, N.; Wang, Y.; He, N.; Sun, J.; and Song, L. 2015. Timesensitive recommendation from recurrent user activities. In Proc. of the Annual Conference on Neural Information Processing Systems (NIPS), 3492-3500.

Du, N.; Dai, H.; Trivedi, R.; Upadhyay, U.; Gomez-Rodriguez, M.; and Song, L. 2016. Recurrent marked temporal point processes: Embedding event history to vector. In Proc. of the ACM SIGKDD International Conference on Knowledge Discovery and Data Mining (KDD), 1555-1564. ACM.

Eichler, M.; Dahlhaus, R.; and Dueck, J. 2017. Graphical modeling for multivariate Hawkes processes with nonparametric link functions. Journal of Time Series Analysis 38(2):225-242.

Etesami, J.; Kiyavash, N.; Zhang, K.; and Singhal, K. 2016. Learning network of multivariate Hawkes processes: a time series approach. In Proc. of the 32nd Conference on Uncertainty in Artificial Intelligence, 162-171. AUAI Press.

Farajtabar, M.; Du, N.; Rodriguez, M. G.; Valera, I.; Zha, H.; and Song, L. 2014. Shaping social activity by incentivizing users. In Proc. of the Annual Conference on Neural Information Processing Systems (NIPS), 2474-2482.

Hall, E. C., and Willett, R. M. 2016. Tracking dynamic point processes on networks. IEEE Transactions on Information Theory 62(7):4327-4346.

Hammond, D. K.; Vandergheynst, P.; and Gribonval, R. 2011. Wavelets on graphs via spectral graph theory. Applied and Computational Harmonic Analysis 30(2):129-150.

Hochreiter, S., and Schmidhuber, J. 1997. Long short-term memory. Neural computation 9(8):1735-1780.

Kalofolias, V.; Bresson, X.; Bronstein, M.; and Vandergheynst, P. 2014. Matrix completion on graphs. arXiv preprint arXiv:1408.1717.

Kingma, D. P., and Ba, J. 2015. Adam: A method for stochastic optimization. In Proc. International Conference on Learning Representations (ICLR).
Kipf, T. N., and Welling, M. 2017. Semi-supervised classification with graph convolutional networks. In Proc. International Conference on Learning Representations (ICLR).

Kurokawa, T.; Oki, T.; and Nagao, H. 2017. Multi-dimensional graph fourier transform. arXiv preprint arXiv:1712.07811.

Lemonnier, R.; Scaman, K.; and Kalogeratos, A. 2017. Multivariate Hawkes processes for large-scale inference. In Proc. of the AAAI Conference on Artificial Intelligence, 2168-2174.

Liniger, T. J. 2009. Multivariate Hawkes Processes. Ph.D. Dissertation, ETH Zurich.

Mallat, S. 1999. A Wavelet Tour of Signal Processing. Academic Press.

Mei, H., and Eisner, J. M. 2017. The neural hawkes process: A neurally self-modulating multivariate point process. In Proc. of the Annual Conference on Neural Information Processing Systems (NIPS), 6754-6764.

Monti, F.; Bronstein, M.; and Bresson, X. 2017. Geometric matrix completion with recurrent multi-graph neural networks. In Proc. of the Annual Conference on Neural Information Processing Systems (NIPS), 3697-3707.

Niepert, M.; Ahmed, M.; and Kutzkov, K. 2016. Learning convolutional neural networks for graphs. In International conference on machine learning, 2014-2023.

Rao, N.; Yu, H.-F.; Ravikumar, P. K.; and Dhillon, I. S. 2015. Collaborative filtering with graph information: Consistency and scalable methods. In Proc. of the Annual Conference on Neural Information Processing Systems (NIPS), 2107-2115.

Sak, H.; Senior, A.; and Beaufays, F. 2014. Long short-term memory based recurrent neural network architectures for large vocabulary speech recognition. arXiv preprint arXiv:1402.1128.

Shang, J., and Sun, M. 2018. Local low-rank hawkes processes for temporal user-item interactions. In Proc. of the IEEE International Conference on Data Mining (ICDM).

Shuman, D. I.; Narang, S. K.; Frossard, P.; Ortega, A.; and Vandergheynst, P. 2013. The emerging field of signal processing on graphs: Extending high-dimensional data analysis to networks and other irregular domains. IEEE Signal Processing Magazine 30(3):83-98.

Wallace, B. 2004. Constrained optimization: Kuhn-tucker conditions.

Wang, Y.; Du, N.; Trivedi, R.; and Song, L. 2016. Coevolutionary latent feature processes for continuous-time user-item interactions. In Proc. of the Annual Conference on Neural Information Processing Systems (NIPS), 4547-4555.

Xu, H.; Wu, W.; Nemati, S.; and Zha, H. 2017. Patient flow prediction via discriminative learning of mutually-correcting processes. IEEE Transactions on Knowledge and Data Engineering 29(1):157-171.

Xu, H.; Farajtabar, M.; and Zha, H. 2016. Learning granger causality for Hawkes processes. In Proc. of the 33rd International Conference on Machine Learning, 1717-1726.

Yang, Y.; Etesami, J.; He, N.; and Kiyavash, N. 2018. Nonparametric hawkes processes: Online estimation and generalization bounds. arXiv preprint arXiv:1801.08273.

Zhou, K.; Zha, H.; and Song, L. 2013. Learning social infectivity in sparse low-rank networks using multi-dimensional Hawkes processes. In Proc. of the 16th International Conference on Artificial Intelligence and Statistics, 641-649. 